\title{
In vitro evaluation of ciclopirox as an adjuvant for polymyxin B against gram-negative bacteria
}

\author{
Kwang-sun Kim ${ }^{1,2}$, Taeyeon $\mathrm{Kim}^{1}$ and Jae-Gu Pan $^{1}$ \\ The Journal of Antibiotics (2015) 68, 395-398; doi:10.1038/ja.2014.164; published online 14 January 2015
}

Polymyxin B is an antibiotic used to treat resistant gram-negative bacterial infections; it was originally isolated from the bacterium Bacillus polymyxa. ${ }^{1}$ Polymyxin B is comprised of related compounds of polymyxins B1, B1-I, B2, B3 and B6; this mixture composition improves the permeability of the compound thus enhancing its bactericidal effects. Polymyxins, including polymyxin B, have been used extensively in topical otic and ophthalmic solutions for decades. ${ }^{2,3}$ Although polymyxins exhibit promising activity against gram-negative bacteria, the nephrotoxicities associated with these compounds have prevented their widespread use in favor of less toxic compounds. However, polymyxins have recently become a more common therapy in the treatment of infections exhibiting extensive multidrug resistance (MDR).

Antibiotic adjuvants or repurposed safe materials may be used to enhance the activity of polymyxins. For example, polymyxin E combined with azithromycin, ceftazidime, imipenem, minocycline, rifampicin or vancomycin shows in vitro synergy against multidrugresistant Acinetobacter baumannii. ${ }^{4,5}$ Polymyxin $\mathrm{B}$ has also been combined with several agents to yield effective combined therapies, ${ }^{6}$ although such combinations may exacerbate the problem of MDR bacteria. Therefore, identification of new synergistic drug combinations may improve treatment efficacy.

Ciclopirox is a synthetic, broad-spectrum antifungal that inhibits dermatophyte growth and has an excellent safety profile. ${ }^{7}$ In over 20 years of clinical use, no fungal resistance has been identified. Therefore, ciclopirox may be repurposed as an anti-human immunodeficiency virus drug, ${ }^{8}$ a protective agent against mitochondrial cell damage, ${ }^{9}$ or a promoter of diabetic wound healing. ${ }^{10}$ Very recently, ciclopirox was also suggested as a repurposed bacteriostatic antibiotic for gram-negative bacteria. ${ }^{11}$ Ciclopirox affects galactose metabolism and alters the composition of lipopolysaccharide (LPS), ${ }^{11}$ a target of polymyxin B. ${ }^{12}$ Thus, ciclopirox may activate polymyxin B function, possibly allowing the reduced use of polymyxin B in treating infections by gram-negative pathogens.

To test our hypothesis, the MICs for ciclopirox and polymyxin B were determined by broth microdilution in a 96-well plate that blocked the visible bacterial growth and the checkerboard assay ${ }^{13}$ was performed to assess the synergy of the two-drug combination against bacterial strains.

The fractional inhibitory concentration index/indices (FICI) were determined from the results of checkerboard assay ${ }^{13}$ after the MICs of each drug for each strain were determined and calculated as follows:

$$
\mathrm{FICI}=\operatorname{MIC}(\mathrm{A} 2) / \mathrm{MIC}(\mathrm{A} 1)+\mathrm{MIC}(\mathrm{B} 2) / \mathrm{MIC}(\mathrm{B} 1),
$$

Where MIC (A2) represented the MIC value of polymyxin B combined with ciclopirox or oxytetracycline, whereas MIC (A1) represented the MIC value of polymyxin B as monotherapy, with the same for MIC (B2) and MIC (B1) in the case of ciclopirox and oxytetracycline. The FICI values were interpreted as follows ${ }^{14} \leqslant 0.5$, synergy; $>0.5$ to $<1$, partial synergy; 1 , addition; $>1$, indifference. The values indicated in Table 1 were isoeffective MIC ranges from five replicated experiments.

The MICs of ciclopirox and polymyxin B for both drug-sensitive Escherichia coli (E. coli) ATCC 25922 and BW25113 strains were 25 $\mu \mathrm{g} \mathrm{ml}^{-1}$ and $0.8 \mu \mathrm{g} \mathrm{ml}^{-1}$, respectively. The checkerboard assay with two-drug combinations was performed against those strains and resulted in the reduction of polymyxin B susceptibility from 0.8 $\mu \mathrm{g} \mathrm{ml}^{-1}$ to the range of $0.2-0.05 \mu \mathrm{g} \mathrm{ml}^{-1}$ (a 4- to 16-fold decrease in the polymyxin B MIC; Figure 1a and Table 1) by increasing concentrations of ciclopirox $\left(3.12-12.5 \mu \mathrm{g} \mathrm{ml}^{-1}\right)$. Synergy was detected at $3.12-6.25 \mu \mathrm{g} \mathrm{ml}^{-1}$ ciclopirox (FICI $=0.312-0.5$ ). Synergy was also detected by growth curve and viability analysis of BW25113 cells treated with ciclopirox and/or polymyxin B (Figures $1 \mathrm{~b}$ and $\mathrm{d}$ ). Ciclopirox $\geqslant 12.5 \mu \mathrm{g} \mathrm{ml}^{-1}$ severely delayed or completely inhibited the growth, but did not result in cell death, thus confirming ciclopirox as a bacteriostatic. ${ }^{11}$ However, the addition of ciclopirox to low-dose polymyxin B conferred bactericidal activity. Thus, ciclopirox may potentiate the bactericidal nature of polymyxin $\mathrm{B}$.

Ciclopirox inhibited the growth of antibiotic-resistant strains and was effective against some gram-negative pathogens; ${ }^{11}$ this synergy might also be effective against MDR isolates and known ciclopiroxtargeted strains. We performed checkerboard assays using MDR E. coli

\footnotetext{
${ }^{1}$ Superbacteria Research Center, Korea Research Institute of Bioscience and Biotechnology (KRIBB), Daejeon, Korea and ${ }^{2}$ Biosystems and Bioengineering, Korea University of Science and Technology, Daejeon, Korea

Correspondence: Dr K-s Kim or Dr J-G Pan, Superbacteria Research Center, Korea Research Institute of Bioscience and Biotechnology (KRIBB), 125 Gwahak-ro, Yuseong-gu, Daejeon 305-806, Korea.

E-mail: sunny06@kribb.re.kr or jgpan@kribb.re.kr

Received 14 August 2014; revised 9 November 2014; accepted 25 November 2014; published online 14 January 2015
} 
Table $1 \mathrm{MIC}$ and $\mathrm{FICl}$ values of $E$. coli, $A$. baumannii and $P$. aeruginosa

\begin{tabular}{|c|c|c|c|c|c|c|}
\hline Species & Strain & $F / C l_{\text {polB/cic }}$ range & $M I C_{p o l B}\left(\mu g m l^{-1}\right)$ & $M I C_{\text {polB/cic }}$ range $\left(\mu g m l^{-1}\right)$ & $M I C_{c i c}\left(\mu g m I^{-1}\right)$ & $M I C_{\text {cic/polB }}$ range $\left(\mu g \mathrm{ml}^{-1}\right)$ \\
\hline \multirow[t]{14}{*}{ E. coli } & ATCC25922 & 0.312 & 0.8 & $0.025-0.2$ & 25 & $3.12-12.5$ \\
\hline & BW25113 & 0.375 & 0.8 & $0.05-0.2$ & 25 & $3.12-12.5$ \\
\hline & MG1655 & 0.312 & 0.8 & $0.05-0.2$ & 25 & $3.12-12.5$ \\
\hline & Keio-galE & 0.375 & 0.4 & $0.05-0.1$ & 25 & $3.12-12.5$ \\
\hline & Keio-galU & 0.5 & 0.2 & $0.025-0.1$ & 25 & $3.12-12.5$ \\
\hline & Keio-rfaB & 0.375 & 0.4 & $0.025-0.1$ & 25 & $3.12-12.5$ \\
\hline & Keio-rfaC & 0.312 & 0.2 & $0.012-0.1$ & 25 & $3.12-12.5$ \\
\hline & Keio-rfaF & 0.375 & 0.2 & $0.025-0.1$ & 25 & $3.12-12.5$ \\
\hline & Keio-rfal & 0.375 & 0.2 & $0.025-0.1$ & 25 & $3.12-12.5$ \\
\hline & Keio-rfaJ & 0.5 & 0.4 & $0.1-0.2$ & 25 & $3.12-12.5$ \\
\hline & Keio-rfaL & 0.625 & 0.4 & $0.1-0.2$ & 25 & $3.12-12.5$ \\
\hline & Keio-rfaP & 0.5 & 0.2 & $0.05-0.1$ & 25 & $3.12-12.5$ \\
\hline & Keio-rfaQ & 0.375 & 0.8 & $0.05-0.2$ & 25 & $3.12-12.5$ \\
\hline & Keio-rfaY & 0.375 & 0.8 & $0.05-0.2$ & 25 & $3.12-12.5$ \\
\hline \multirow[t]{3}{*}{ MDR E. coli } & CCARM 1 A074 & $0.25-0.5$ & 0.8 & $0.05-0.1$ & 25 & $3.12-6.25$ \\
\hline & CCARM 1 A078 & $0.25-0.5$ & 0.8 & $0.05-0.1$ & 25 & $3.12-12.5$ \\
\hline & CCARM 1 A645 & $0.375-0.5$ & 0.4 & $0.05-0.1$ & 25 & $3.12-12.5$ \\
\hline A. baumannii & ATCC 15150 & $0.375-0.5$ & 1.6 & $0.012-0.4$ & 25 & $3.12-12.5$ \\
\hline \multirow[t]{2}{*}{ MDR A. baumannii } & CCARM 12001 & $0.375-0.5$ & 1.6 & 0.4 & 25 & $3.12-12.5$ \\
\hline & CCARM 12003 & $0.375-0.5$ & 1.6 & 0.4 & 25 & $3.12-12.5$ \\
\hline$P$. aeruginosa & PA01 & $0.531-1.0$ & 1.6 & $0.4-0.8$ & 100 & $3.12-75.0$ \\
\hline
\end{tabular}

Abbreviations: Cic, ciclopirox; FICI, fractional inhibitory concentration index/indices; MDR, multidrug resistance; PolB, polymyxin B.

The $\mathrm{FICl}_{\text {polB/cic }}$ indicates the values at the most synergistic combination between polymyxin $\mathrm{B}$ and ciclopirox. MIC $\mathrm{C}_{\text {polB }}$ and $\mathrm{MIC}_{\text {cic }}$ indicate the $\mathrm{MIC}$ values of polymyxin $\mathrm{B}$ and ciclopirox, respectively. $\mathrm{MIC}_{\text {polB/cic }}$ and $\mathrm{MIC}_{\mathrm{cic} / \mathrm{polB}}$ represent the MIC values of polymyxin B and ciclopirox in the presence of ciclopirox or polymyxin B except the point of MIC values of single compound, respectively. All $\mathrm{FICl}$ values were determined from Figure 1 and Supplementary Figures 1-3. All keio collections (http://www.shigen.nig.ac.jp/ecoli/strain/) were confirmed by PCR amplification with gene-specific primers (Supplementary Table 1) and DNA sequencing.

isolates and a drug-sensitive A. baumannii (ATCC 15150) strain and observed synergy between polymyxin B and ciclopirox, with FICI values of $0.25-0.5$ in E. coli-MDR (Figure 1e) and $0.375-0.5$ for A. baumannii (Figure 1f). The synergy of combined polymyxin B and azithromycin, imipenem or rifampicin is highly strain dependent, ${ }^{5}$ however, we found that the synergy of polymyxin B and ciclopirox was the same in all the A. baumannii strains regardless of drug-resistant status (FICI $=0.375-0.5$; Figure 1f). Thus, the in vitro synergy of ciclopirox and polymyxin B seemed to be more generalized for gramnegative bacteria than other previously tested combinations.

To investigate whether efficient ciclopirox targeting is required for synergy, we selected Pseudomonas aeruginosa PAO1, an inefficient target for ciclopirox. ${ }^{11}$ The MIC of ciclopirox in PAO1 was 100 $\mu \mathrm{g} \mathrm{ml}^{-1}$, much higher than in E. coli or A. baumannii (MICs: 25 $\mu \mathrm{g} \mathrm{ml}^{-1}$; Figure $1 \mathrm{~g}$ and Table 1). Meanwhile, the combination of ciclopirox and polymyxin $\mathrm{B}$ reduced the MICs in PAO1 by two to fourfold (3.12-75 $\mu \mathrm{g} \mathrm{ml}^{-1}$ ciclopirox and $0.4 \mu \mathrm{g} \mathrm{ml}^{-1}$ polymyxin B); however, partial synergy or addition $(\mathrm{FICI}=0.531$ to 1.0 ) was observed at these concentrations (Figure $1 \mathrm{~g}$ and Table 1). These data showed that effective ciclopirox targeting seems to be required for synergy with polymyxin B.

Because polymyxin B acts in synergy with multiple antibiotics rather than a single specific antibiotic against gram-negative bacteria ${ }^{6,15,16}$ it may questioned whether ciclopirox also shows the synergy with multiple antibiotics rather than specific to polymyxin B. Checkerboard assays were performed to determine the effect of ciclopirox in combination with five classes of antibiotics including cephalothin (cephalosporin), gentamicin (aminoglycosides), levofloxacin (quinolone), meropenem (carbapenem) and minocycline (tetracycline). None of the tested antibiotics showed synergy or adjuvant effects; an increase in ciclopirox concentration did not improve E. coli susceptibility to the antibiotic mixtures (Supplementary Figure 1), suggesting the interaction of ciclopirox with polymyxin B is specific.

Polymyxin B-containing products such as Terramycin, an ointment that also contains oxytetracycline, are used worldwide. Such mixtures are used to eliminate gram-negative bacterial infections, but prolonged use of oxytetracycline greatly increases the frequency of tetracyclineresistant bacteria in humans and feed animals. ${ }^{17}$ Drug resistance leads to more serious bacterial infections. Therefore, we suggest polymyxin B could be alternately combined with ciclopirox and oxytetracycline for improved killing of gram-negative bacteria. To compare the efficacies of both mixtures, the synergy between oxytetracycline and polymyxin B was measured by checkerboard assay with E. coli ATCC 25922 (Figure 1h). The MIC of oxytetracycline was $0.25 \mu \mathrm{g} \mathrm{ml}^{-1}$ and the MIC of polymyxin B was reproducibly decreased by twofold with $0.125 \mu \mathrm{g} \mathrm{ml}^{-1}$ oxytetracycline. However, no synergy was detected between polymyxin $\mathrm{B}$ and oxytetracycline at this concentration $(\mathrm{FICI}=1.0$; addition $)$. Therefore, ciclopirox plus polymyxin B provided better synergy than oxytetracycline plus polymyxin $\mathrm{B}$. Therefore, the addition of ciclopirox to oxytetracycline/polymyxin B or the combination of ciclopirox and polymyxin B might provide an alternate to Terramycin.

Ciclopirox modifies the composition of the O-antigen, the outermost layer of the LPS structure; ${ }^{11}$ thus, we suspect that the absence of $\mathrm{O}$-antigen may inhibit the synergistic effect. This possibility was tested by checkerboard assays for MG1655, a strain lacking O-antigen, ${ }^{18}$ and the O-antigen producing E. coli ATCC25922, which showed synergy was not eliminated by the absence of $\mathrm{O}$-antigen $(\mathrm{FICI}=0.312-0.5$; Supplementary Figure 2 and Table 1). Therefore, O-antigen modification by ciclopirox does not increase susceptibility to polymyxin B.

Products of galE gene rescued bacterial growth at restrictive ciclopirox concentration and gene knockouts in $g a l U, r f a I, r f a B, r f a C$ or rfaQ make cells susceptible to ciclopirox. ${ }^{11}$ These genes are 
a
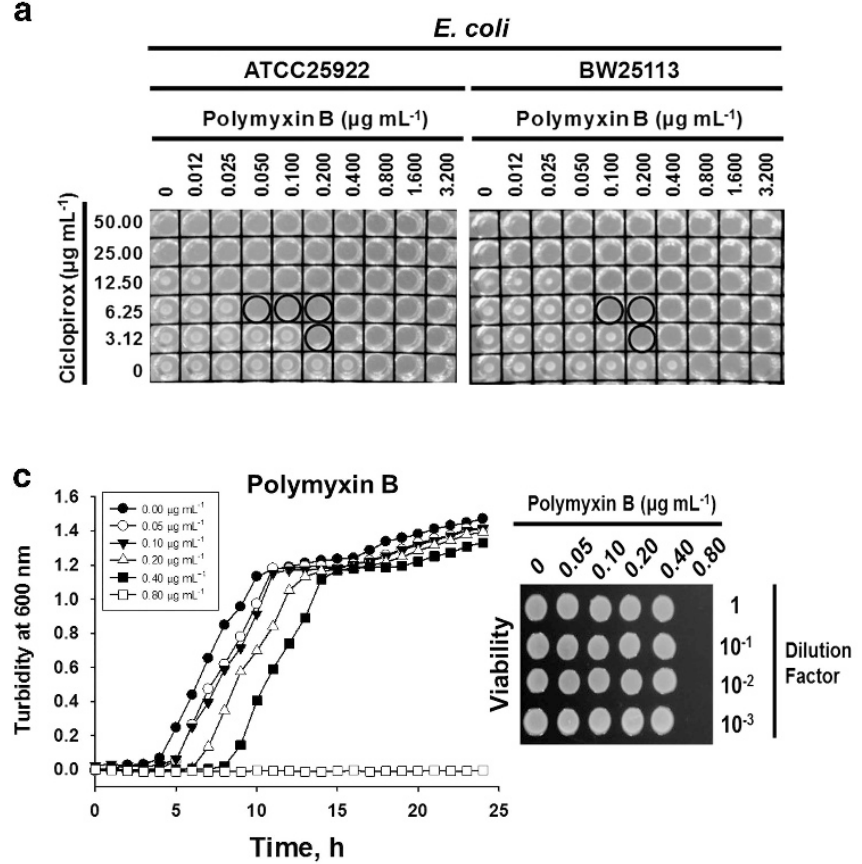

b
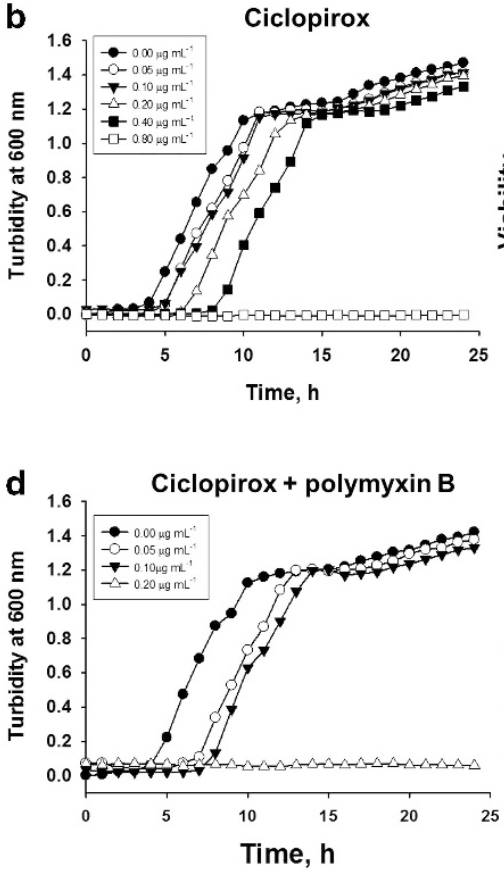

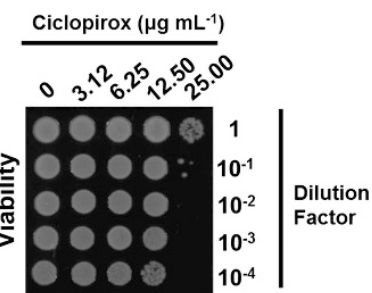

Polymyxin B $\left(\mu \mathrm{g} \mathrm{mL}^{-1}\right)$

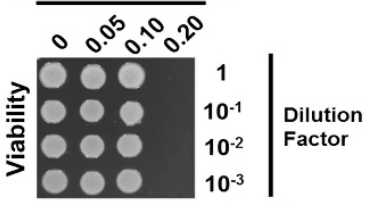

with Ciclopirox $3.12 \mu \mathrm{g} \mathrm{mL}^{-1}$ e

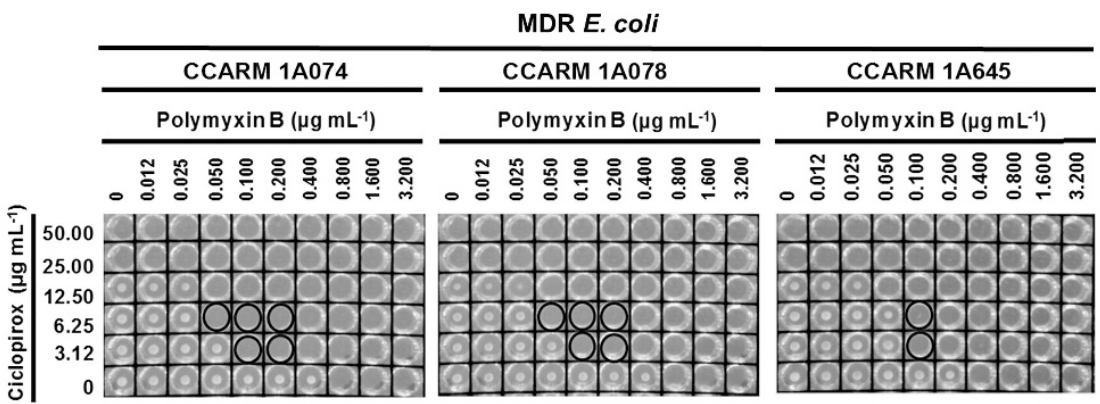

f

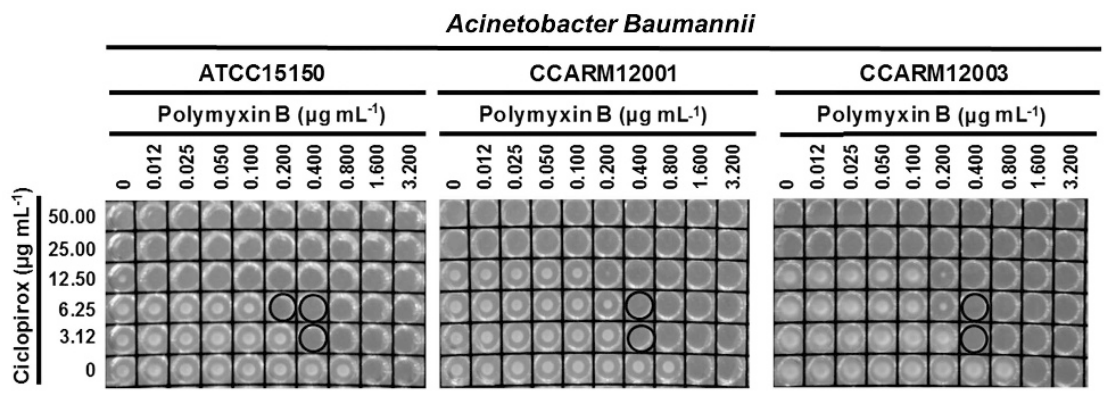

g

Pseudomonas aeruginosa (PA01)

Polymyxin B ( $\left.\mu \mathrm{g} \mathrm{mL}^{-1}\right)$

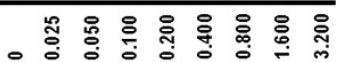

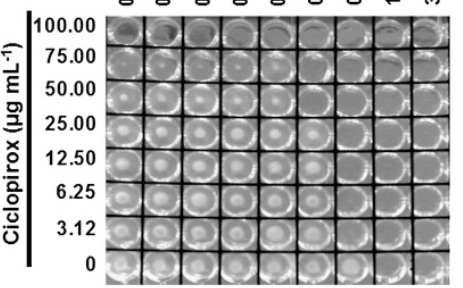

h

ATCC25922

Polymyxin B $\left(\mu \mathrm{g} \mathrm{mL}^{-1}\right)$

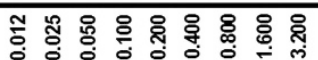

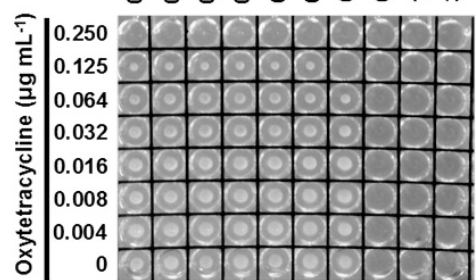

Figure 1 Synergy of ciclopirox and polymyxin B. (a) Synergistic effects of ciclopirox and polymyxin B in E. coli cells. MIC and synergistic patterns in ATCC25922 and BW25113 are shown. (b-d) Effect of ciclopirox, polymyxin B or ciclopirox/polymyxin B on the growth and cell viability of E. coli BW25113 cells. One of the representatives from three independent experiments. (e) Synergistic effects of ciclopirox and polymyxin B in MDR E. coli strains. Clot images of CCARM 1A074, CCARM 1 A078 and CCARM 1A645 strains are shown. (f, g) Conservation of synergy. Clot images are shown for A. Baumannii strains ATCC 15150, CCARM 12001 and 12003, and P. aeruginosa PAO1. (h) Evaluation of oxytetracycline as a synergistic agent to polymyxin B. The checkerboard assays were performed using E. coli ATCC 25922 strain. Synergistic combination is indicated with black circle. Absence of a clot indicates the bactericidal effect of antibiotics in mono- or combination-therapy.

associated with galactose modification and LPS biosynthesis. Therefore, we evaluated the role of specific gene products and pathways in the synergy between ciclopirox and polymyxin B by checkerboard assays and the Keio collection of E. coli and its parental strain BW25113 (Supplementary Figure 3 and Table 1). The genes were divided into groups on the basis of the FICI values at the strongest synergic combinations: (i) non-regulatory genes galE, $r f a B, r f a C, r f a F$, $r f a I, r f a Q$ and $r f a Y$ (FICI $=0.312-0.375)$; (ii) partially effective genes galU, $r f a J$ and $r f a P(\mathrm{FICI}=0.5)$; and (iii) a partial synergy gene $r f a L$ $(\mathrm{FICI}=0.625)$, which is the first gene in the synthesis of the outer core 
of the LPS structure. Thus, the $r f a L$ product is likely the most effective modulator of synergy. In addition, knockout of $r f a L$ affected the susceptibility to polymyxin B. Our data suggest that efficient synergy between polymyxin B and ciclopirox requires modified sugar utilization LPS structure between component change through ciclopirox and disruption of structure by polymyxin B (Supplementary Figure 4).

Both polymyxin B and ciclopirox have been also used as topical fungal agents. Therefore, the synergy of ciclopirox and polymyxin B might also be useful as a new antifungal formulation, similar to the current use of fluconazole to reduce polymyxin B toxicity. ${ }^{19}$ Fluconazole is a widely used topical drug for treating superficial and systemic fungal infections; it has been used to reduce the high MIC of polymyxin B to clinically relevant concentrations in the treatment of Aspergillus fumigatus, ${ }^{19}$ thus reducing the side effects of polymyxin B. The administration of ciclopirox, a compound with an excellent safety profile and no fungal resistance in more than 20 years of clinical use, ${ }^{11}$ warranted treatment of phase I hematologic malignant patients. ${ }^{20}$ Alone, it does not produce severe toxicity or adverse effects in humans. For instance, dose-limiting gastrointestinal toxicities in patients with hematologic malignancies were not observed with oral administration of $80 \mathrm{mg} \mathrm{m}^{-2}$ ciclopirox olamine four times daily; $10-40 \mathrm{mg} \mathrm{m}^{-2}$ once daily administration did not produce doselimiting toxicity. The effective dose of ciclopirox for human fungal or bacterial infection in combination with polymyxin B is much lower than the dose used to treat hematologic malignancy. In addition, $<5 \%$ of treated patients showed mild local reactions following skin and vaginal application of ciclopirox. ${ }^{7}$ Therefore, ciclopirox may be more beneficial in combination with polymyxin B for the treatment of fungal infections.

In conclusion, we are the first to report ciclopirox with polymyxin B as a new synergistic combination with the ability to modulate the resistance of MDR E. coli and A. baumannii. This provides a potential alternative to the current limited use of polymyxin B in treating gramnegative bacterial infection. Detailed mechanistic studies including identification of genes modulated by ciclopirox or the combination of ciclopirox and polymyxin B are required to understand the synergy between these drugs. Clinical trials are also needed to support the use of this drug combination in the practical setting.

\section{CONFLICT OF INTEREST}

The authors declare no conflict of interest.

\section{ACKNOWLEDGEMENTS}

We thank the National BioResource Project (NIG, Japan) for their support of the distribution of the Keio collection. This work was supported by the Korea
Research Institute of Bioscience \& Biotechnology (KRIBB) initiative programs, Korea.

1 Falagas, M. E. \& Kasiakou, S. K. Colistin: the revival of polymyxins for the management of multidrug-resistant gram-negative bacterial infections. Clin. Infect. Dis. 40, 1333-1341 (2005).

2 Nakajima, S. Clinical use of colimycin F otic solution. Jibiinkoka 37, 693-697 (1965).

3 Ohzawa, R. The use of colimycin ear drops. Jibiinkoka 37, 585-590 (1965).

4 Gordon, N. C., Png, K. \& Wareham, D. W. Potent synergy and sustained bactericidal activity of a vancomycin-colistin combination versus multidrug-resistant strains of Acinetobacter baumannii. Antimicrob. Agents Chemother. 54, 5316-5322 (2010).

5 Tripodi, M. F., Durante-Mangoni, E., Fortunato, R., Utili, R. \& Zarrilli, R. Comparative activities of colistin, rifampicin, imipenem and sulbactam/ampicillin alone or in combination against epidemic multidrug-resistant Acinetobacter baumannii isolates producing OXA-58 carbapenemases. Int. J. Antimicrob. Agents 30, 537-540 (2007).

6 Wareham, D. W. \& Bean, D. C. In vitro activities of polymyxin B, imipenem, and rifampin against multidrug-resistant Acinetobacter baumannii. Antimicrob. Agents Chemother. 50, 825-826 (2006).

7 Subissi, A., Monti, D., Togni, G. \& Mailland, F. Ciclopirox: recent nonclinical and clinical data relevant to its use as a topical antimycotic agent. Drugs 70, 2133-2152 (2010)

8 Hoque, M. et al. Inhibition of HIV-1 gene expression by Ciclopirox and Deferiprone, drugs that prevent hypusination of eukaryotic initiation factor 5A. Retrovirology 6, 90 (2009).

9 Lee, S. J. et al. Ciclopirox protects mitochondria from hydrogen peroxide toxicity. Br. J. Pharmacol. 145, 469-476 (2005).

$10 \mathrm{Ko}, \mathrm{S} . \mathrm{H}$. et al. Antimycotic ciclopirox olamine in the diabetic environment promotes angiogenesis and enhances wound healing. PLoS One 6, e27844 (2011).

11 Carlson-Banning, K. M. et al. Toward repurposing ciclopirox as an antibiotic against drug-resistant Acinetobacter baumannii, Escherichia coli, and Klebsiella pneumoniae. PLOS ONE 8, e69646 (2013).

12 Huttner, B. et al. Drugs of last resort? The use of polymyxins and tigecycline at US veterans affairs medical centers, 2005-2010. PLoS One 7, e36649 (2012).

13 Elipoulos G. M. \& Moellering R. C. in Antibiotic in Laboratory Medicine 4th edn (ed. Lorian, V.) 330-396 (William and Wilkins Baltimore, MD, 1996).

14 Dong, X. et al. In vitro activities of rifampicin, colistin, sulbactam and tigecycline tested alone and in combination against extensively drug-resistant Acinetobacter baumannii. J. Antibiot. 67, 677-680 (2014).

15 Yoon, J., Urban, C., Terzian, C., Mariano, N. \& Rahal, J. J. In vitro double and triple synergistic activities of polymyxin $B$, imipenem, and rifampin against multidrugresistant Acinetobacter baumannii. Antimicrob. Agents Chemother. 48, 753-757 (2004)

16 Wareham, D. W. \& Bean, D. C. In-vitro activity of polymyxin B in combination with imipenem, rifampicin and azithromycin versus multidrug resistant strains of Acinetobacter baumannii producing OXA-23 carbapenemases. Ann. Clin. Microbiol. Antimicrob 5, 10 (2006)

17 Tadesse, D. A. et al. Antimicrobial drug resistance in Escherichia coli from humans and food animals, United States, 1950-2002. Emerg. Infect. Dis. 18, 741-749 (2012).

18 Zhai, B. et al. Polymyxin B, in combination with fluconazole, exerts a potent fungicidal effect. J. Antimicrob. Chemother. 65, 931-938 (2010).

19 Minden, M. D. et al. Oral ciclopirox olamine displays biological activity in a phase I study in patients with advanced hematologic malignancies. Am. J. Hematol. 89 363-368 (2014).

20 Stevenson, G. et al. Structure of the 0 antigen of Escherichia coli K-12 and the sequence of its rfb gene cluster. J. Bacteriol. 176, 4144-4156 (1994).

Supplementary Information accompanies the paper on The Journal of Antibiotics website (http://www.nature.com/ja) 